\title{
Nondestructive wood discrimination: FTIR - Fourier Transform Infrared Spectroscopy in the characterization of different wood species used for artistic objects
}

\author{
Maria Crista Buoso \\ INFN-Legnaro National Laboratories, Viale dell'Università 2, 35020 Legnaro (PD), Italy \\ buoso@lnl.infn.it \\ Mario De Poli \\ INFN-Legnaro National Laboratories, Viale dell'Università 2, 35020 Legnaro (PD), Italy \\ mdepoli@lnl.infn.it \\ Peter Matthaes \\ Museo d'Arte e Scienza, Via Q. Sella 4, 20121Milano, Italy \\ pr@museoartescienza.com \\ Luca Silvestrin \\ Department of Physics and Astronomy, University of Padova, via Marzolo 8, 35131 Padova, Italy \\ luca.silvestrin@pd.infn.it \\ Demetre Zafiropoulos \\ INFN-Legnaro National Laboratories, Viale dell'Università 2, 35020 Legnaro (PD), Italy \\ zafiropoulos@lnl.infn.it
}

Published 1 September 2016

\begin{abstract}
Wooden artifacts represent a significant component of past cultures. Successful conservation of wooden artifacts depends on the knowledge of wood structure and types. It is critical that conservators know the category of wood that they are treating in order to successfully conserve it. Recently, vibrational spectroscopy has been successfully applied to determine the chemical structure of wood and to characterize wood types. FTIR (Fourier Transform Infrared) is a useful nondestructive or micro-destructive analytical technique providing information about chemical bonding and molecular structure. Its application in the discrimination between softwoods (conifers) and hardwoods (broad-leafs) has already been reported. The aim of the present study was to investigate the potential of FTIR as a tool for the discrimination between different wood types belonging to the same genus. Three different hardwood species, namely poplar (Populus spp), lime (Tilia spp) and birch (Betula spp), were investigated by means of FTIR spectroscopy. The woods were first inspected using a light microscope to certify the wood essence types through micrographic and morphoanatomical features. The FTIR spectra in the $4000 \mathrm{~cm}^{-1}$ to $450 \mathrm{~cm}^{-1}$ region were recorded using a Perkin-Elmer Spectrum 100 spectrometer. To enhance the qualitative
\end{abstract}

This is an Open Access article published by World Scientific Publishing Company. It is distributed under the terms of the Creative Commons Attribution 3.0 (CC-BY) License. Further distribution of this work is permitted, provided the original work is properly cited. 
interpretation of the IR spectra, second derivatives of all spectra were calculated using the Spectrum software to separate superimposed bands and to extract fine spectral details. To obtain a comprehensive characterization, the essences under investigation were also analyzed by means of Raman Spectroscopy. Clear differences were found in the spectra of the three samples confirming FTIR to be a powerful tool for wood type discrimination.

Keywords: Spectroscopic method; FTIR, Wood discrimination.

\section{Introduction}

Over centuries of human history, different species of wood have been widely used in the execution of artefacts. Each geographic region favored certain wood types not only from technical, economic, and practical factors but also from the peculiarities of the artisan tradition. Moreover, only a limited number of them have been utilized in the production of timber for artistic purposes. In the past, birch was the most used specie for the wooden panels in byzantine icon painting. ${ }^{1}$ Artists from northern Germany and Holland preferred oak, while those in southern Germany favored woods such as spruce and lime. In Spain, poplar, pine and occasionally walnut were commonly used while, in Italy, linden and poplar were the most adopted. ${ }^{2}$

In the cultural heritage domain, when dealing with historic wooden objects, accurate identification of wood species can be of paramount importance since it enables appropriate conservation treatments and adequate choice of materials to be used in the restoration of historical wooden objects. ${ }^{3}$ In a modern context, forgeries can be identified from improper wood type. Vibrational spectroscopy, especially IR spectroscopy, offers a large variety of applications in the study of art objects and has been successfully used both to elucidate the wood chemical structure and to characterize wood types.

Fourier Transform Infrared spectroscopy (FTIR) has proved to be a very useful nondestructive or micro-invasive analytical technique. Its application to the discrimination of softwood (conifers) from hardwood (broad-leafs) has already been established. ${ }^{4,5,6}$

The goal of the present work is to ascertain whether FTIR can be successfully used to discriminate between three hardwood essences widely used as an artistic medium: poplar (Populus spp), lime (Tilia spp) and birch (Betula spp). These wood types can be hardly discriminated by a simple bulk optical inspection. A blind experiment of anatomical identification has been carried out by IVALSA-CNR trees and timber Institute San Michele all'Adige (TN) Italy, in order to perform a comprehensive validation of the method. ${ }^{7}$

\section{Materials and Methods}

\subsection{Materials}

For each species, five trees almost of the same age were chosen for sampling. Only healthy individuals with no apparent injury were used. Ten blocks $(10 \mathrm{~cm} \times 8 \mathrm{~cm} \times 5 \mathrm{~cm}$, length $\mathrm{x}$ width $\mathrm{x}$ thickness respectively) have been cut out from the fresh wooden boards. 
Experimental samples have been conditioned for three weeks in a climatic chamber, in a dry environment at room temperature, to unify the moisture distribution within blocks.

\subsection{Microscopic Analysis}

The wood block samples were sectioned using a sliding microtome with freezing plate to produce transverse, radial-longitudinal and tangential-longitudinal sections. The sections were then examined using a reflection light Olympus BX51 optical microscope fitted with an Olympus TH4-200 reflected light source. In the study of wood surfaces, the transverse, radial and tangential sections have specific characteristics and appearance to the observation, but most important is the transverse section because of the high number of observable characteristics.

\subsection{FTIR Analysis}

From each block, three samples of wood powder were taken from different locations on the surface. Powder samples of a few milligrams were obtained using a low rotating speed electric drill with a milling-bit of less than $3 \mathrm{~mm}$ diameter. Each sample was taken fairly deep in the wood after removing the first $2-3 \mathrm{~mm}$ from the surface. This standard sampling protocol is adopted to avoid thermal modifications in the wood structure and possible contaminations coming from the surface.

The wood powder was then carefully mixed with spectroscopic grade $\mathrm{KBr}$ and pelletized under a pressure of 8 tons. The content of wood in the pellets ranged from 10 to $15 \mathrm{mg}$ approximately.

FTIR spectra were measured using the FT-NIR Perkin-Elmer Spectrum 100 spectrometer of the Spectroscopy Laboratory at the Museo d'Arte e Scienza. ${ }^{8}$ The measured spectral range was chosen between $450 \mathrm{~cm}^{-1}$ and $4000 \mathrm{~cm}^{-1}$ with a resolution of $4 \mathrm{~cm}^{-1}$, standard conditions for such a measurement. In order to increase the signal-tonoise ratio, each final spectrum was the average of 16 single acquisition scans. The Spectro software package has been used both for data acquisition and data analysis.

\section{Results}

\subsection{Anatomical identification}

Wood is composed of different kinds of cells with different arrangements for each type of tree. The observation of the diagnostic anatomical characteristics allows wood type identification in the different samples. ${ }^{6}$ The results from anatomical and structural analysis, as reported from IVALSA-CNR Trees and Timber Institute, are reported below.

\subsubsection{Sample no. 1 (Fig. 1)}

In the transverse section, the semi-ring porous structure is clearly visible with rather distinct growth ring boundaries, depending on pore size transition from earlywood to latewood. According to some growth conditions, these boundaries can be rather 
indistinct. The pores are sparsely scattered, solitary, in radial multiples of 2 to 4 , and in clusters. Pore size decreases gradually to small pores in the latewood without clear delineation between earlywood and latewood. The sparse and diffuse apotracheal parenchyma is occasionally arranged in uniseriate, discontinuous terminal bands.

From the radial and tangential sections, the homogeneous rays appear to be both uniseriate and multiseriate, occasionally with square marginal cells. Ray-vessel pits are numerous and extremely small. The perforation plates are scalariform with 10 to 15 (sometimes up to 25) bars. Moreover, the presence of libriform fibres is confirmed while fibre-tracheids are absent.
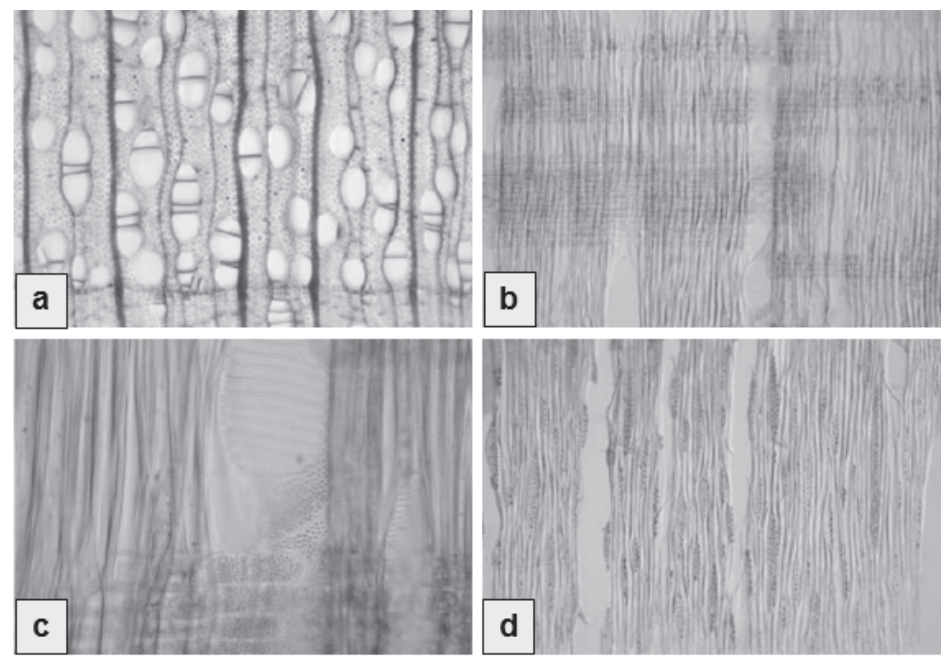

Fig. 1. Microscopic sections of Betula spp: $\mathrm{a}$-Transverse section; $\mathrm{b}$ - Radial section; $\mathrm{c}$ - Radial section, scalariform perforation plate; $\mathrm{d}$ - Tangential section.

\section{Diagnosis: Betula spp.}

\subsubsection{Sample no. 2 (Fig. 2)}

In the transverse section, the semi-ring porous structure with oblate pores solitary, in radial groups, or in radial rows of 2 to 3 multiples, is clearly visible. Growth ring boundaries were rather distinct depending on pore size transition from earlywood to latewood. The sparse and diffuse apotracheal parenchyma is occasionally arranged in uniseriate, discontinuous terminal bands.

In the radial section, the homogeneous rays are rare and with square marginal cells. The perforation plates are simple, and cross-field pitting appeared as areolar oopores. The presence of libriform fibres is confirmed while, fibre-tracheids are absent. The tangential section reveals the presence of longitudinal uniseriate rays. 

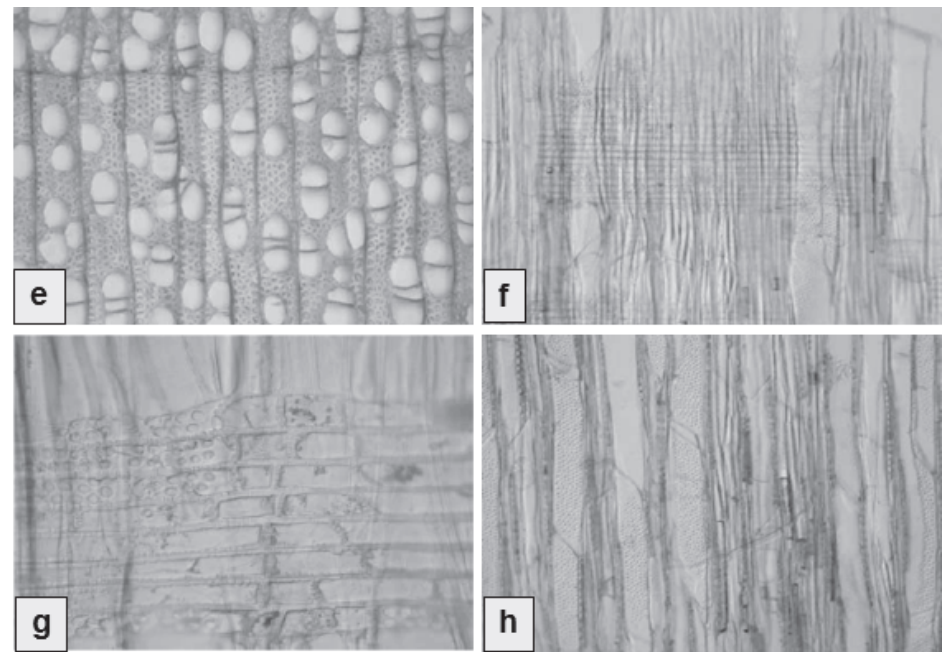

Fig. 2. Microscopic sections of Populus spp; e - Transverse section; $\mathrm{f}$ - Radial section; $\mathrm{g}$ - Radial section, Homogeneous rays; $\mathrm{h}$ - Tangential section.

\section{Diagnosis: Populus spp.}

\subsubsection{Sample no. 3 (Fig. 3)}

In the transverse section, the diffuse semi-ring porous structure is clearly visible. The slightly polygonal pores are often in radially oriented files and clusters. The growth ring boundaries are marked by 2 to 3 rows of thick-walled radially flattened cells and distinctly flaring rays. The apotracheal parenchyma is generally arranged in short, uniseriate, oblique to tangential bands.

From the radial and tangential sections, the homogeneous rays appear to be both uniseriate and multiseriate, occasionally with square marginal cells. The uniseriate rays are composed of upright cells. Simple perforation plates and conspicuous spiral thickenings in the vessels and are clearly evidenced. The ground tissue is predominantly composed of libriform fibres, seldom of fibre-tracheids. The Ray-vessel pits are numerous and small. 

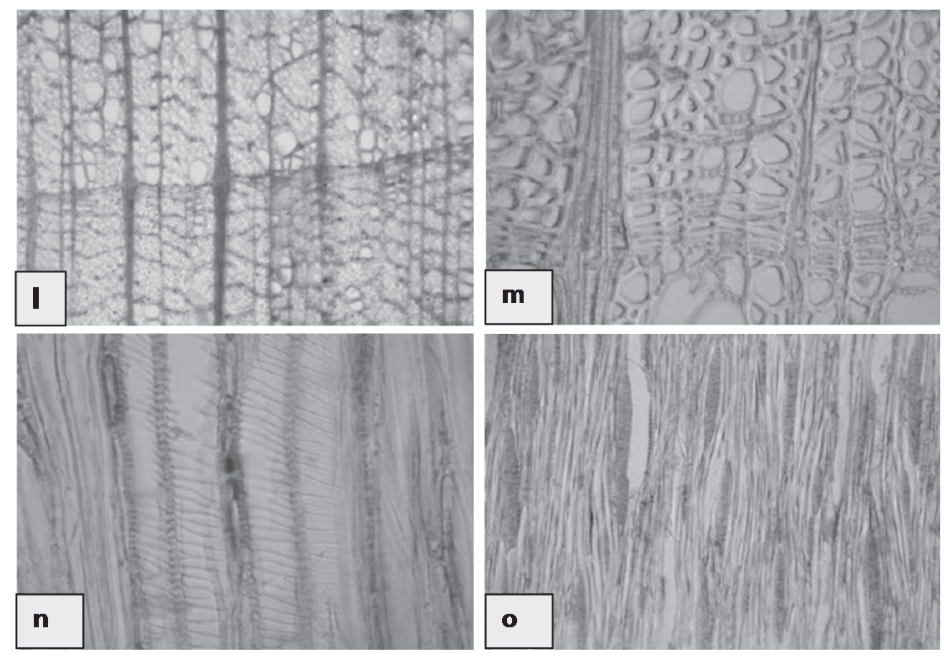

Fig. 3. Microscopic sections of Tilia spp; 1 - Transverse section; $\mathrm{m}$ - Wall thickness of fibers; $\mathrm{n}$ - Radial section; o - Tangential section.

Diagnosis: Tilia spp.

\subsection{FTIR}

The FTIR spectra show the same basic structure: a strong broad O-H stretching at 3300$3600 \mathrm{~cm}^{-1}, \mathrm{C}-\mathrm{H}$ stretching at $2800-3000 \mathrm{~cm}^{-1}$ and several distinct peaks in the fingerprint region between 450 and $1800 \mathrm{~cm}^{-1}$. Most of these bands have contributions from both carbohydrates (cellulose and hemicellulose) and lignin. Figure 4 shows a typical FTIR spectrum of Populus spp along with the fingerprint region.

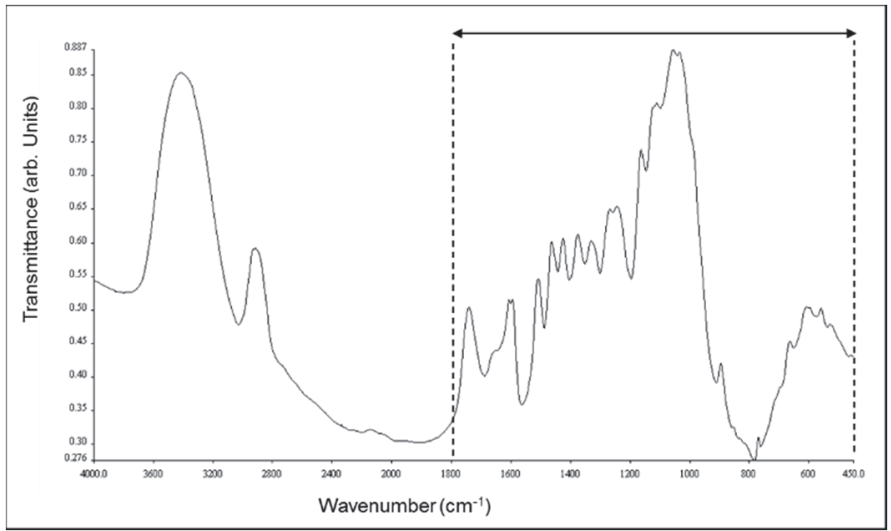

Fig. 4. FTIR spectrum of Populus spp wood. On the right, the fingerprint region is marked.

Comparing the FTIR spectra of the three wood species in Fig. 5, a clear differentiation between Populus spp and the other two wood essences can be observed. 
The spectrum of Populus presents characteristic peaks at $1607 \mathrm{~cm}^{-1}$ and $771 \mathrm{~cm}^{-1}$ that are not present in the spectra of Betula spp and Tilia spp. Moreover, the peak between $1300-1200 \mathrm{~cm}^{-1}$ shows differences, as well as a lower intensity of the peak at 1740-1745 $\mathrm{cm}^{-1}$. The last peaks cannot be used as fingerprints for the identification of Populus as they do not belong exclusively to the absorption domains of this type of wood, but they can be associated to different species.

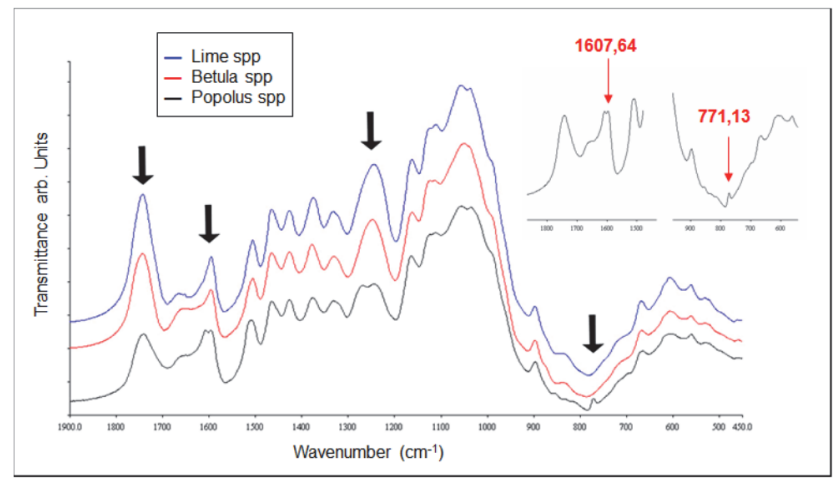

Fig. 5. Comparison between the FTIR spectra of the three different wood essences and the fingerprint regions of Populus spp.

While differences between the poplar and the other two are obvious, the differentiation between the lime and birch woods is more difficult in the direct spectra. More information can be extracted using the second derivative of the spectra. The application of the second derivative transformation of FTIR spectroscopic data allows the identification of small differences that are decisive to distinguish the two types of wood, as can be seen in Fig. 6. Again, the most important regions were those between $1800-1690 \mathrm{~cm}^{-1}$ and $1300-1200 \mathrm{~cm}^{-1}$.

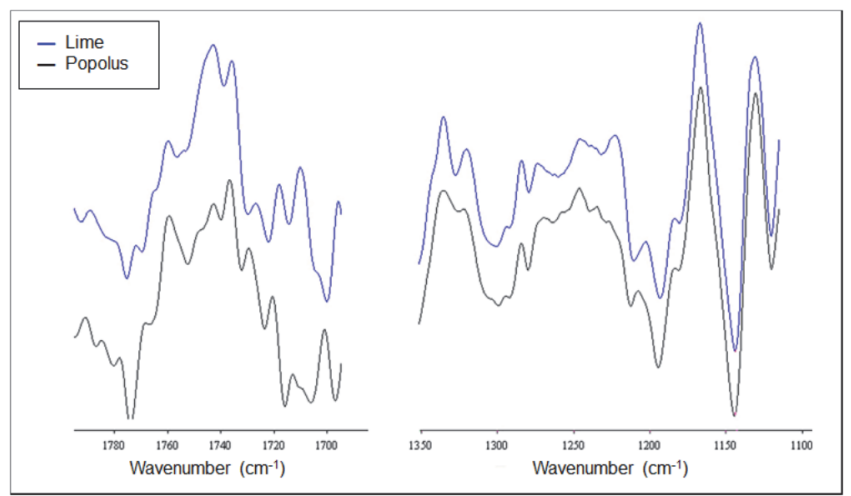

Fig. 6. The second derivative spectra for Lime and Populus for the wavenumber regions of $1800-1690 \mathrm{~cm}^{-1}$ and $1350-1100 \mathrm{~cm}^{-1}$. 
To confirm the applicability of the method, FTIR measurements were performed on poplar, lime and birch samples coming from 15 art objects of different typologies and with different geographical origin and age. This allowed the restriction of the poplar value of absorption characteristic peaks in a rather narrow range: between 770 and 772 $\mathrm{cm}^{-1}$ for the peak at lower wave numbers and $1606-1608 \mathrm{~cm}^{-1}$ for the peak at higher wave numbers. The small interval of $2 \mathrm{~cm}^{-1}$ confirms the relevance of these peaks to characterize the essence of poplar.

\section{Conclusions}

Three different species utilized in wooden artifacts have been selected for their unique mechanical, visual, exotic or symbolic properties. FTIR has proved its capability to discriminate wood species and can be applied in a huge number of situations. In conservation or restoration of historic furniture, monuments or other wooden artworks, the prior knowledge of the original wood type can be essential for selecting appropriate methods or materials. The comparison between FTIR spectra from the poplar, linden and birch samples showed characteristic peaks for the poplar samples, while for the linden and birch, no macroscopic differences were highlighted. In order to obtain a better discrimination between linden and birch, the second derivative mode was applied. Significant differences among the two species became evident. Future work plans will include the analysis and the characterization of other wood essences. Since totally noninvasive wood identification is an indisputable need for many wooden art objects, preliminary tests using UATR (Universal Attenuated Total Reflectance) measurements on macroscopic wood samples were also performed. The obtained preliminary data gave too poor results, while UATR measurements on natural wood powder led to very promising evidences.

\section{References}

1. L. Ouspensky and V. Lossky, The Meaning of Icons (St. Vladimir Seminary Press, New York, 1999).

2. The Structural Conservation of Panel Paintings: Proc. Symposium at the J. Paul Getty Museum, eds. K. Dardes and A. Rothe, (Getty Conservation Institute, Los Angeles, 1995), http://hdl.handle.net/10020/gci_pubs/panelpaintings

F. Gruchow et al., Preservation Science 6, 145 (2009), http://www.morana-rtd.com/epreservationscience/TOC.html

3. P. Niemz, Methods of non-destructive wood testing, in 2008 Proc. Int. Conf. on Wood Science for Conservation of Cultural Heritage, ed. J. Gril (Braga, Portugal, 2008), p. 163, http://www.fupress.com/Archivio/pdf\%5C4502.pdf.

4. S. Tsuchikawa et al., Journal of Wood Science 49, 29 (2003).

5. S. S. Kelley et al., Wood Sci. Technol. 38, 257 (2004).

6. Microscopic Wood Anatomy of Central European species. Web-based identification key: http://www.wsl.ch/dienstleistungen/datensaetze/woodanatomy/index_EN.

7. G. Matthaes, in 30th Annual Meeting of American Institute for Conservation of Historic and Artistic Works (Miami, Florida, USA, 2002) 